\title{
Testing Oil Saturation Distribution in Migration Paths Using MRI
}

\author{
Jianzhao Yan ${ }^{1}$, Xiaorong Luo ${ }^{1}$, Weimin Wang $^{2}$, Fang Chen ${ }^{3}$, Renaud Toussaint ${ }^{4}$, \\ Jean Schmittbuhl ${ }^{4}$, Guy Vasseur ${ }^{5}$, Likuan Zhang ${ }^{1}$
}

1. Key Laboratory of Petroleum Resources Research, Institute of Geology and Geophysics, Chinese Academy of Science, Beijing, 100029 China.

2. School of Electronics Engineering and Computer Science, Peking University, Beijing, 100871 China.

3. State Key Laboratory of Magnetic Resonance and Atomic and Molecular Physics, Wuhan Institute of Physics and Mathematics, Chinese Academy of Sciences, Wuhan, 430071 China.

4. IPGS(Institute de Physique du Globe de Strasbourg), CNRS(Centre National de la Recherche Scientifique), University of Strasbourg, 5 rue Descartes, Strasbourg, 67000 France.

5. Sisyphe, UMR7619, University Pierre et Marie Curie, Paris Cedex 05, 75252 France.

Abstract: Magnetic Resonance Imaging (MRI) method allows to observe the distribution of different fluids in situ in porous media, and to measure oil and water saturation. Although this technique has great advantages compared to others, there remains large space for assessing the method and improving the accuracy of measurement. Using MRI, the oil secondary migration paths are scanned to measure the saturation distribution during the laboratory experiments. The resulting map can be calibrated using a device with the same pore structure as the probed sample and fully saturated with oil. This device is scanned with the probed sample at the same time in order to calibrate the saturation. The Spin-echo multi-slices sequence (SEMS) is adopted for MRI to ensure that the oil saturation in migration paths is accurately measured. The relevant spatial resolution of the mapping is defined according to the concept of REV (representative elementary volume). The oil saturation resulting from data obtained using different image formats are compared and the resulting saturation evaluation is compared to direct bulk saturation measurements. This comparison demonstrates that the calculated MRI oil saturation using DICOM image format is quite accurate, with a relative error less than $2 \%$.

Key Words: MRI; Oil Saturation; Calibration; DICOM; Migration Path

\section{Introduction}

Physical experiment is an important means of observing the hydrocarbon migration phenomenon and studying the migration mechanism (Dembicki and Anderson, 1989; Catan et al., 1992; Tukunaga et al., 2000; Zeng and Wang, 2003; Zhang et al., 2003; Zhang et al., 2004). For interpreting these experiments, it is important to evaluate the oil saturation occurring in the migration channel. Moreover the oil saturation gives an important basis for the 
quantitative evaluation of the migration losses and for the estimation of potential reservoir (Hirsch and Thompson, 1995; Luo, et al., 2004; Luo et al., 2007; Luo et al., 2008).

Many conventional methods of measuring oil saturation, including distillation, chromatography (Tang and Cai, 2007) and water washing method (Dembicki and Anderson, 1989), have been proposed as listed in table 1. These methods are essentially destructive, i.e. samples are destroyed to measure the oil saturation in the porous medium so that the oil saturation can not be dynamically monitored. Besides, all results measured with conventional methods are average oil saturation (Chen et al., 1992; Nicula et al., 1998), which makes difficult to assess the spatial distribution of oil in the porous medium (Baldwin et al., 1989). So in the recent decades, scientists have developed some new non-destructive technologies, such as optical techniques and ray attenuation method in order to test the spatial distribution of fluid saturation in biphasic flows in porous media. Optical techniques, including light reflection method (LRM) (Van and Sykes, 1998; Fishman et al., 1998; O'Carroll et al., 2004) and light transmission method (LTM) (Hoa et al., 1981; Darnault et al., 1998; Darnault et al., 2001; Conrad et al., 2002; Bob et al., 2008) are low cost, easy to fulfill, but only apply to quasi2D transparent media. Compared with conventional light sources, both Gamma rays and X rays have a relatively strong penetrability and apply to opaque media such as soil and rock (Høst-Madsen and Jensen, 1992; Liu et al., 1992; Ursin, 1992; Illangasekare, et al., 1995; Imhoff et al., 1996; Bsavaraj et al., 1997; Dicarlo et al., 2000; Hill et al. 2002; Oostrom et al., 2003; Oostrom et al., 2005). But as for optical methods, they are half-space measuring methods, and only apply to 2D plate models with low thickness. Computer tomography (CT) methods using Gamma or X ray attenuation can realize actual 3D local measurement of non-transparent medium without limitation of medium shapes (Shama et al., 1997; Mogensen et al., 2005; Rangel-German et al., 2006). However, since the absorption and attenuation of $\mathrm{X}$ ray mainly depend on the local density of the porous media (i.e. matrix + fluid) but not on fluids themselves, it is hard to distinguish between fluids. Therefore the CT Gamma or X-ray method is not well adapted to observe the oil and water in porous medium (Edelstein et al., 1998; Li et al., 2007).

Compared with the above methods, MRI method has huge advantages in testing oil saturation. First, as a nondestructive and noninvasive method, MRI has a high testing precision ${ }^{12}$ and can be used to monitor the saturation change during the experimental process; second, MRI provides a much better contrast to distinguish the fluids between them than CT because it detects the fluids in the porous medium directly; third, MRI does not require a particular shape, realizing 3D spatial quantitative measurement in a real sense; and last, MRI allows to see through opaque rocks, unlike optical methods only suitable for Hele Shaw cells.

Many researchers have measured oil saturation by detecting $H^{+}$density using MRI since 1990s (Baldwin et 
al., 1989; Mandava et al., 1990; Chardaireriviere and Roussel, 1991; Chen et al., 1992; Enwere and Archer, 1992; Chang et al., 1993; Mogensen et al., 2005). Washburn (2010) adopted Na element to conduct MRI to distinguish between the water phase and the oil phase, and tested the distribution of water-phase saturation with MRI. Most of the tests, however, were carried out in one-dimensional space. Graue et al. (2001) have given the oil distribution in 2D MRI slices, but the test principle and image processing were not described.

Secondary migration of oil and gas in basins is an extremely heterogeneous process (McNeal, 1961; Harms, 1966; Smith, 1966; Berg, 1975; Schowalter, 1979), and heterogeneous path is found even in homogeneous porous media as long as the gravitational or viscous contrast between the two fluids destabilizes the invasion process (Frette et al., 1997; Méheust 2002; Luo et al., 2004; Løvoll et al., 2004; Toussaint et al., 2005). Therefore, real-time measurement of shape features of the migration path and the change of its interior oil saturation is an essential basic work for research of hydrocarbon migration mechanism. Miao et al. (2004) and Zhou et al. (2005) have adapted the MRI method to define oil saturation during the oil migration process. They calibrated the measurements using pure oil filled in a small glass tube as calibration, but they didn't consider the possible matrix effect: this effect results in a significant difference between in-pore oil and pure oil estimate from transverse relaxation rate and the overall accuracy should take into account this effect

This paper focuses on the technique of using MRI to measure oil saturation in heterogeneous oil clusters inside a porous medium. Experiments are conducted in a glass tube initially saturated with water where oil migrates, while another glass tube filled with glass beads and fully saturated with oil is used as the calibration. This allows measuring the oil saturation and its distribution inside the path of different type of patterns. The paper also makes a comparative analysis of differences in oil saturation calculated according to data sources of different image formats, and validates the techniques by comparison with independently measured global saturation results.

\section{Measurement Principle}

In the experiments, the SEMS was used to scan the samples and the relaxation process of MRI signal can be described as (Mandava et al., 1990):

$$
M\left(\omega, T_{E}\right)=M_{0}(\omega) \exp \left(-\frac{T_{E}}{T_{2}}\right)\left(1-\exp \left(-\frac{T_{R}}{T_{1}}\right)\right)
$$

in which, $\omega$ is the resonance frequency, $M$ is the observed magnetic intensity, $M_{0}$ is the inherent magnetic intensity, $T_{E}$ is the echo time, $T_{R}$ is the repeat time, $T_{1}$ is the longitudinal relaxation time, and $T_{2}$ is the transverse relaxation time. $T_{1}$ and $T_{2}$ are dependent on the properties of matter and the surrounding 
environment; $T_{E}$ and $T_{R}$ can be set in the MRI sequence. If $T_{R}>>T_{1}$ and $T_{E}<<T_{2}$, the observed magnetic intensity is close to the inherent one:

$$
M\left(\omega, T_{E}\right) \approx M_{0}(\omega)
$$

The gray value of the obtained images is in direct proportion to the proton density:

$$
M_{0}(\omega)=k N\left(H^{+}\right)
$$

which is the basis for oil saturation testing with MRI (Edelstein et al., 1988; Chen et al., 1992).

During the scan of the probed sample with MRI, if a calibration is carried out aside with a known oil saturation $\left(S^{\prime}\right)$, then for the calibration sample, as well:

$$
M^{\prime}\left(\omega^{\prime}, T_{E}^{\prime}\right) \approx M_{0}^{\prime}\left(\omega^{\prime}\right)
$$

in which, $M^{\prime}$ and $M_{0}^{\prime}$ are the observed magnetic intensity and inherent magnetic intensity of the calibration respectively. Calling the oil saturation of the probed sample and of the calibration sample respectively as $S$ and $S^{\prime \prime}$, Considering $\omega=\omega^{\prime}$ and $T_{E}=T_{E}^{\prime}$, the oil saturation in the samples can be decided by the oil saturation in the calibration:

$$
S=\frac{M\left(\omega, T_{E}\right)}{M^{\prime}\left(\omega, T_{E}\right)} S^{\prime}
$$

$$
\text { time. In the oil and water two-phase displacement experiments, } \mathrm{H}^{+} \text {in both oil and water can incur MRI signal, }
$$
but affected by the molecular structure, the relaxation rates of two MRI signals are different, namely, the relaxation of $\mathrm{H}^{+}$spin in water molecules is quicker than that in oil. Under addition of water-soluble $\mathrm{Mn}^{2+}$ in water, the relaxation time of $\mathrm{H}^{+}$in water molecule will become even shorter, while that in oil remains unchanged (Chang et al., 1993). Figure 1 describes the dependence of the transverse relaxation time of manganese water solution on the $\mathrm{Mn}^{2+}$ concentration, in experimental conditions (i.e. in pores between piled glass beads of $0.6 \mathrm{~mm}-0.8 \mathrm{~mm}$ grain size). As can be seen on the figure 1, the relaxation times fall dramatically with $\mathrm{Mn}^{2+}$ for small concentrations. When $\mathrm{Mn}^{2+}$ concentration above $700 \mathrm{mg} / \mathrm{L}$, water relaxation times become much shorter than those of oil ( $T_{1}$ and $T_{2}$ for oil are respectively $1024 \mathrm{~ms}$ and $868 \mathrm{~ms}$, whereas for manganese water $T_{1} \sim 7 \mathrm{~ms}$ and $T_{2} \sim 3.5 \mathrm{~ms}$ ). Under such conditions, it is possible to isolate the MRI signal of oil and neglect the one of the 
manganese water solution. Setting the $\mathrm{T}_{\mathrm{E}}$ to values interval between manganese water and oil transverse

relaxation time, i.e. $T_{2 w}<<T_{E}<<T_{20}$, and keeping $T_{R}$ above the longitudinal relaxation time of oil, $T_{10}<<T_{R}$, allows to neglect the contribution of $H^{+}$in water molecule to MRI signal, and to record the inherent magnetic intensity from the oil molecules .

Oil saturation is measured by comparing MRI signal intensity of the probed sample with that of the calibration.

\section{Effect of the porous matter structure of the calibration sample on the determination of the oil relaxation time}

The accurate measurement of oil saturation requires oil relaxation time in calibration to be the same as in the probed sample.

As pointed out by Kenyon (1992), the transverse relaxation of fluid in pore medium is governed by free relaxation, surface relaxation and diffusion relaxation:

$$
\frac{1}{T_{2}}=\frac{1}{T_{2 b u l k}}+\rho_{2} \frac{s}{v}+\frac{D\left(\gamma G T_{E}\right)^{2}}{12}
$$

in which, $T_{2 \text { bulk }}$ is the transverse relaxation time of free fluid, $\rho_{2}$ is the transverse relaxation intensity, $s / v$ is the specific surface, $D$ is the magnetic diffusion coefficient, $\gamma$ is the gyromagnetic ratio, and $G$ is the field intensity gradient. The first item on the right side of equations (6) represents the transverse relaxation rate of the free fluid, far from liquid/solid boundaries; the second term represents the surface relaxation rate, while the third term represents the magnetic diffusion relaxation rate. As can be seen in the equation, compared with a pure fluid and its free relaxation time, the fluid in porous medium is partly bounded to the solid, affected by surface relaxation mechanisms, and the transverse relaxation time will be shortened. In general, the diffusion relaxation mechanism does not cause significant differences between the calibration and testing sample in transverse relaxation time, and if short enough $T_{E}$ is adopted to conduct the MRI, the influence of diffusion relaxation can be neglected.

In the present experiment, we use oil as displacement phase fluid and a porous medium fully saturated with oil as a calibration sample, $T_{2}=T_{2}^{\prime}$ and $S^{\prime}=100 \%$, so the equation (5) can be deducted as:

$$
S=\frac{M\left(\omega, T_{E}\right)}{M^{\prime}\left(\omega, T_{E}\right)}
$$


The specific surface of the porous medium is a decreasing function of the pore radius (Pape et al., 2009),

namely, the pore radius will affect the transverse relaxation of fluid in the porous media. To quantitatively evaluate the effect of surface relaxation on the relaxation time, we tested the transverse relaxation time of pure free oil and of the oil in pores of glass bead piling body of different grain sizes (Table 2), by adopting the multi-echo spin echo sequence (CPMG) ( Wamg et al., 2008). It can be seen from Table 2 that, with the decrease of the glass bead size, the transverse relaxation time shortens clearly. In order to eliminate this influence, the sample and the calibration should be filled with the glass beads with same size.

\section{Sample Making and Testing Method and Flow}

We conducted secondary migration experiments in a glass tube of length $55 \mathrm{~cm}$ and inner diameter $2.6 \mathrm{~cm}$. To build the migration model, we adopted the wet filling method (Hou et al., 2004): first, one end of the glass tube was plugged with a rubber plug and filled with water, next a mixture of glass beads and water was poured into the tube, which was beaten and shaken from time to time. Eventually when the top surface of the filled glass beads was $1 \mathrm{~cm}$ away from the top end, the tube was plugged with another rubber plug, which led to get the model fully saturated with water. The diameter of the glass beads used was $0.6-0.8 \mathrm{~mm}$. Oil was dyed red so that its movement could be traced directly optically. The calibration tube used in the experiment, with the same specification as the tube model, was also filled with the same size glass beads using the wet filling method, but it was saturated with dyed oil.

Dyed oil was injected into the glass tube from the top end while the water was expelled through the bottom plug. Injection was stopped when the height of the oil column reached $24 \mathrm{~cm}$ and the glass tube was inverted upside down. Oil migrated upwards driven by the buoyancy and the migration pattern could be observed visually as narrow rising strings (Luo et al., 2004). After 10 minutes, the migration front almost reached the top end of the glass tube and the tube was moved horizontally in the middle of the MRI testing chamber. The calibration sample was put closely and parallel to the tested sample.

The MRI scanner used in the experiment is Wandong $1.5 \mathrm{~T}$ superconducting MRI scanner at a proton frequency of $63.89 \mathrm{MHz}$. According to the measured $T_{1}$ and $T_{2}$ values of oil in the piling glass bead pores, we set $T_{R}$ to $6000 \mathrm{~ms}$ and set $T_{E}$ as the minimum value allowed by the meter: $15 \mathrm{~ms}$. We also set the slice thickness ( $T$ ) as $2 \mathrm{~mm}$ and interval between slices $(G)$ as $0.2 \mathrm{~mm}$. It took 52 minutes to complete once scan.

\section{NMR Image Processing}

Since NMR imaging results from measurements of weak signals, the effect of noise should be taken into 
consideration when calculating oil saturation with MRI. Figure 2A is the MRI slice along the horizontal direction, perpendicular to the vertical migration direction, and Figure $2 \mathrm{~B}$ is the gray level distribution along the red line marked in the slice. In Figure 2A, the white disk in the upper left corresponds to the calibration sample; the white part in the lower right corresponds to the migration path; and the blue circle marks the position of to-be-measured glass tube in the image. As seen from Figure 2B, the gray level in the path in not homogeneous, and even outside of the main migration path, the gray value is not zero, which is commonly called noise. Comparing Figure $2 \mathrm{~A}$ with Figure $2 \mathrm{~B}$, it can be seen that the noise in non-oil area inside the glass tube is consistent with the noise outside the tube, indicating that the NMR signal of manganese water has been completely shielded.

The background noise level can be estimated by measuring the gray value of image in an oil-empty area (Chen et al., 1992), defined as a rectangular area besides the testing sample: From the gray values of each pixel point in such area, the background level is computed as the average value $B_{n}$ :

$$
B_{n}=\frac{1}{j} \sum_{i=1}^{j} g_{i}
$$

in which $\mathrm{j}$ is number of pixels in the rectangular area, and $g_{i}$ is the gray level of the pixel of index i. $B_{n}$ is about 28 and far smaller than the signal of oil. In order to lower the effect of the noise, equation (7) can be modified as:

$$
S=\frac{M\left(\omega, T_{E}\right)-B_{n}}{M^{\prime}\left(\omega, T_{E}\right)-B_{n}}
$$

Attention must be paid to the image format when computing the saturation according to MRI. The default image format of MRI is DICOM (Digital Imaging and Communications in Medicine) 3.0 format. The DICOM image output by Wandong superconducting MRI meter has a 12 digits dynamics, i.e. 4096 gray levels. Commonly used image processing software, such as ACDSee and Photoshop, can not support DICOM image format. To facilitate the image display and processing, windowing display technology is often used to convert DICOM format into BMP format (or TIF format) (Nakashima and Kamiya, 2007; Groesel et al., 2009). This procedure normally implies to choose a zone in the DICOM image, used for a gray level conversion into the BMP or TIF format. As will be illustrated hereafter, caution has to be paid on the choice of this window in practice, since this affects the type of transform and the quality of the quantitative saturation assessment obtained by the whole treatment chain.

The conversion from DICOM format into BMP format is usually partially linear (appendix). The setting of window width and level can directly influence the relative distribution of gray values at each pixel point of the converted BMP format image.

Two sets of window and level were compared when converting DICOM image into BMP image. In a first test, -7 - 
the window and level were adjusted to make the migration path clearest and to generate the BMP format image (Figure 3A). In a second test, image format was converted from DICOM to BMP while the window width covering the whole signal ranges (Figure 3B). The oil saturation distribution was calculated according to Figure $3 \mathrm{~A}$ and Figure 3B respectively (Figure 4A and Figure 4B). In Figure 4A and 4B, the average oil saturations in migration path are $48.15 \%$ and $35.13 \%$ respectively; it is clear that, the selection of windows directly influences the calculation results of oil saturation. By comparing the oil saturation distribution diagram calculated in BMP format (Figure 4) with that calculated directly in DICOM format (Figure 5), it can be found that, only when the window covers the whole pixel interval, both diagrams can match well. This choice should thus always be preferred for a quantitative saturation estimate - even though it may reduce the brightness and contrast of the converted image, which is not the most favorable for the direct observation of the structure of the migration path (Figure 3).

\section{Assessment of the MRI saturation measurement precision in a porous medium model}

To check the reliability and determine the accuracy of the oil saturation measurement with MRI, a comparison is done of the total oil mass calculated from the determined saturation distribution with the total oil mass injected into the model.

The glass tube was first fully filled with water, with two ends closed by rubber plugs. Then water was poured out and its volume $v_{t}$ was measured. The glass tube was refilled with manganese water with a $\mathrm{Mn}^{2+}$ concentration of $700 \mathrm{mg} / \mathrm{L}$, and the mixture of glass beads of $0.6-0.8 \mathrm{~mm}$ grain diameter and manganese water was poured into the glass tube. The top end of the glass was blocked with a rubber plug, and total mass of the model $m_{1}$ was weighed. A certain amount of oil was injected into the glass tube model, and then the glass tube and the calibrating tube underwent a complete MRI scanning at the same time. Calculating the average oil saturation in migration path of each slice and the sectional area of migration path with the DICOM format image, the oil volume of such slice can be calculated as:

$$
v_{s}=\bar{S}_{s}^{*} A_{s} * T * \phi
$$

in which, $\bar{S}_{s}$ is the average oil saturation of such slice in migration path, $A_{s}$ is the sectional area of migration path, $T$ the slice thickness and $\phi$ is the porosity of the model. Though the part between two slices can not be MRI scanned, since the interval between slices is much smaller than the slice thickness, the oil volume in the gaps between slices can be interpolated from the two neighboring slices: 


$$
v_{G}=\frac{\left(A_{s}+A_{s+1}\right)}{2} * \frac{\left(\bar{S}_{s}^{-}+S_{s+1}^{-}\right)}{2} * G * \phi
$$

with $n$ is the total number of the slices, the oil volume in the whole model is obtained as:

$$
v_{o}=\sum_{s=1}^{n} V_{s}+\sum_{G=1}^{n-1} V_{G}
$$

In the next step, an increment of oil volume is further injected, and the whole measurement chain described above is determined. A total of nine complete scans of the experiments are conducted. After the completion MRI tests, the glass beads were taken out from the glass tube model, washed tidily and dried, after that the total mass $m_{2}$ of the glass beads, glass tube and rubber plugs is measured. Then the volume of manganese water in the glass tube model is determined as:

$$
v_{p}=\frac{m_{1}-m_{2}}{\rho_{M n}}
$$

in which, $\rho_{M n}$ is the mass density of a $700 \mathrm{mg} / \mathrm{L}$ concentrated manganese water. The porosity of the glass tube model is calculated from these volumes as $\phi=0.36$.

A direct comparison of the calculated total oil volume with the actually injected oil volume, in Figure 6, demonstrates a very good match.. The average relative error between the direct measurements and MRI derived measurements, among the nine groups of data, is only $1.57 \%$.

Since the type of oil pattern may vary from stable (piston-like) to unstable (finger) as a function of injection velocity (Tukunaga et al., 2000; Luo et al., 2004), it is important to discuss the accuracy of saturation measurements as a function of migration pattern. Finger pattern and respectively piston migration pattern were observed when oil was injected from the bottom of the glass tube at injection flux of respectively $1.0 \mathrm{ml} / \mathrm{min}$ and $4.0 \mathrm{ml} / \mathrm{min}$. Figure 7 and Figure 8 show the oil saturation in one slice of the finger and piston migration path respectively. For finger migration path, the calculated total oil volume is $32.4 \mathrm{ml}$ whereas the actually injected oil volume is $33 \mathrm{ml}$ so that the average relative error between them is $1.82 \%$. For piston migration path, the calculated total oil volume is $78.7 \mathrm{ml}$ whereas the actually injected oil volume is $78 \mathrm{ml}$ separately, so that the average relative error between them is $0.9 \%$.

\section{Discussion}

Like the porosity and permeability, the saturation is an average physical parameter depending on the measurement scale. Chardaireriviere (1993) adopted high-field MRI to improve the spatial resolution when testing 
fluid saturation, with the minimum pixel of $100 \mu \mathrm{m} \times 100 \mu \mathrm{m} \times 50 \mu \mathrm{m}$, but it does not mean that the highest spatial resolution is the best choice to adopt. If the spatial resolution was high enough, the local oil saturation should in principle anywhere be either 0 or $100 \%$ according to the presence of either water or oil in a pore, which should reflect the smallest details of the structure of the oil distribution at small scales in the pores, but this choice leads to very noisy measurements. The proper measurement scale can be decided according to the concept of REV (Bear, 1972). On the one hand, the REV shall be small enough to fully reflect the change of spatial distribution of oil saturation on the path, and on the other hand, the REV also shall be large enough to eliminate the effect of the smallest scales, i.e. the effect of the structure of each pore on the determined oil saturation. Figure 9 is the distribution of signal intensity of the calibrating tube fully saturated with oil by testing with different spatial resolutions at the same place. The saturation is homogeneous in the calibration tube, and the choice of the measurement scale should allow recovering an almost homogeneous signal at the REV scale, erasing the small scale variations of the porosity. It can be seen on Figure 9 that, when the resolution is too high, e.g. the pixel reaches $0.25 \mathrm{~mm} * 0.25 \mathrm{~mm}$, the signal intensity fluctuates dramatically with the change of spatial position; when the pixel reaches $1 \mathrm{~mm} * 1 \mathrm{~mm}$, the signal intensity basically reaches stability and the influence of porous media structure is eliminated. Consequently, in the tests mentioned in this paper, all MRI resolutions are set as $1 \mathrm{~mm} * 1 \mathrm{~mm}$. It should be pointed out that the proper measurement scale should be determined again by a similar procedure if the size of the glass beads is changed. The intensity of the signal increases while the pixel size increasing because larger pixel covers more oil.

Another question is whether the vertical migration path does occur in the columns during the scan time when the tubes lie on their long side in the MRI chamber . Catalan et al. (1992) computed the minimum height $\left(h_{\min }\right)$ of oil column for migration as:

$$
h_{\min }=\frac{\left(P_{d r}-P_{i m b}\right)}{g\left(\rho_{w}-\rho_{o}\right)}
$$

$$
P_{d r(w / o)}=P_{d r(w / a)} \frac{\sigma_{(w / o)} \cos \theta}{\sigma_{(w / a)}}
$$

$$
P_{i m b(w / o)}=P_{i m b(w / a)} \frac{\sigma_{(w / o)} \cos \theta}{\sigma_{(w / a)}}
$$

in which $P_{d r}$ and $P_{i m b}$ are drainage and imbibition capillary pressures respectively, $w / a$ means water-air system, $w / O$ means the water-oil system, the interface tension of water-oil system $\sigma_{(w / o)}$ and water-air system 
$\sigma_{(w / a)}$ are $0.0289 \mathrm{~N} / \mathrm{m}$ and $0.072 \mathrm{~N} / \mathrm{m}$, the contact angle for water-oil system $\theta$ is $30^{\circ}, \rho_{w}=1014 \mathrm{~kg} / \mathrm{m}^{3}$ is the

density of manganese water, $\rho_{o}=792 \mathrm{~kg} / \mathrm{m}^{3}$ is the density of oil, and $g=9.80 \mathrm{~m} / \mathrm{s}^{2}$ is the gravity acceleration. The drainage and imbibition capillary pressures were $1079 \mathrm{~Pa}$ and $638 \mathrm{~Pa}$ respectively for the water-air system when the glass bead diameter was $0.72 \mathrm{~mm}$ (Catalan et al., 1992). The average diameter used in our experiment is very close $0.72 \mathrm{~mm}$. So the drainage and imbibition capillary pressures (1079Pa and 638Pa respectively) can be used to predict the minimum height of the oil column for migrating for our experiment.

$$
\begin{aligned}
& P_{d r(w / o)}=1079 \times 0.0289 \times \cos \left(30^{\circ}\right) / 0.072=375.1 \mathrm{~Pa} \\
& P_{i m b(w / o)}=638 \times 0.0289 \times \cos \left(30^{\circ}\right) / 0.072=221.8 \mathrm{~Pa}
\end{aligned}
$$

and from equation (14):

$$
h_{\min }=(375.1-221.8) /[9.8 \times(1014-792)]=0.07 \mathrm{~m}
$$

While the glass tube was placed horizontally, the maximum height of oil column is equal to the inner diameter of the tube $(2.6 \mathrm{~cm})$, which is obviously smaller than $h_{\min }$. Consequently, oil should not migrate significantly during the MRI scan when the glass tube is horizontal, and the MRI pictures should hardly be affected by such fluid flow.

For more precision of the saturation measurements, the MRI signals of manganese water and oil can be compared quantitatively. In the porous medium, while $T_{1}$ and $T_{2}$ of oil is $1024 \mathrm{~ms}$ and $868 \mathrm{~ms}, T_{1}$ and $T_{2}$ of manganese water are just $6.88 \mathrm{~ms}$ and $3.45 \mathrm{~ms}$. When $T_{R}$ and $T_{E}$ are equal to $6000 \mathrm{~ms}$ and $15 \mathrm{~ms}$ respectively, according to equation (1), for manganese water, we can get:

$$
M^{w}=0.0129 * M_{0}^{w}
$$

in which, $M^{w}$ and $M_{0}^{w}$ are the observed magnetic and inherent magnetic intensity and of manganese water respectively. For oil, we also can get:

$$
M^{o}=0.9801 * M_{0}^{o}
$$

in which, $M^{\circ}$ and $M_{0}^{o}$ are the observed magnetic and inherent magnetic intensity and of oil respectively. The inherent magnetic intensity of $\mathrm{H}^{+}$is decided by the abundance of hydrogen atoms. In water $\left(\mathrm{H}_{2} \mathrm{O}\right)$, the abundance of hydrogen atoms is $11.11 \%$; in oil $\left(\mathrm{CH}_{3}\left(\mathrm{CH}_{2}\right)_{n} \mathrm{CH}_{3}, \mathrm{n}=8 \sim 16\right)$, the abundance of hydrogen atoms is from $14.17 \%$ to $15.28 \%$, which is slightly larger than the abundance of hydrogen atoms in water. So the 
difference of inherent magnetic intensity between water and oil is small. Thus, comparing equation (17) with equation (18), the MRI signal of water is much smaller than the one of oil, and can be neglected. The result of quantitative analysis is consistent with the observation (Figure 2). If the signals of water want to be shielded, the following condition is needed:

$$
T_{2}^{w}<<T_{E}<<T_{2}^{o}
$$

in which, $T_{2}^{w}$ and $T_{2}^{o}$ are the transverse relaxation time of water and oil respectively.

\section{Conclusions}

Compared with the testing methods of fluid saturation commonly used before, MRI can make accurate measurements of oil saturation and its 3D spatial distribution in porous media. Considering various kinds of possible influencing factors, a procedure of measure was developed, and it was established that the oil saturation calculated by MRI has a relatively high precision with the average relative error less than $2.0 \%$.

When adopting the gray values of MRI to calculate oil saturation, it is better to directly use the original image in DICOM format; or when the windowing technology is used to convert DICOM format into BMP format to calculate oil saturation, the window must cover the gray value distribution interval of the whole image. Besides the image format, the measurement resolution also should be selected carefully according to the concept of REV.

MRI technology can be used not only to observe the structural form of migration path, but also to quantitatively analyze the distribution of oil saturation, and it has broad application potentials in experiments of secondary migration and even fluid flow in porous medium. For actual rocks, the probed sample itself can be used as the calibration. After the rock saturated fully with oil being scanned by MRI as the calibration signal, it scanned again when saturated oil is being displaced by water, while keeping the testing conditions and rock's position unchanged. The saturation distribution can be computed by comparing the MRI signal of the two scans. The detailed determination of the saturation distribution in actual rocks is the subject of ongoing work..

\section{Acknowledge}

This study was supported mainly by Chinese National Major Fundamental Research Developing Project (2011CB201105), Chinese National Natural Science Foundation (41102079), and Chinese National Key Scientific and Technological Project (2011ZX08005-004), partly University of Strasbourg, France.

\section{Appendix}

DICOM is a set of communication format of imaging in medicine formulated by ACR (American College of Radiology) and NEMA (National Electrical Manufacturers Association), aiming to unify the interface calibration 
1 for equipment of different manufacturers. DICOM image is a kind of gray-level image, and the image file contains two parts --- file header and data element. It may be 8 digits, 12 digits or 16 digits. Windowing display technology is often used to convert a DICOM format into BMP format (or TIF format). The so-called windowing display is to specify a gray-level window, and according to the following equation, convert linearly the image in such window area into the largest display scope of the display device, and the image data higher or lower than the limits of window are set as the maximum or the minimum, respectively (Zhang et al., 2003).

$$
G(V)=\left\{\begin{array}{lc}
0 & V<c-\frac{w}{2} \\
\frac{g_{m}}{w}\left(V+\frac{w}{2}-c\right) & c-\frac{w}{2} \leq V \leq c+\frac{w}{2} \\
g_{m} & V>c+\frac{w}{2}
\end{array}\right.
$$
the maximum value of image gray value. 


\section{References}

1. Baldwin, B.A., Yamanashi, W.S., 1989. Detecting fluid movement in reservoir core with medical NMR imaging techniques, SPE reservior Eng. 4(2), 207-212.

2. Bear, J., 1972. Dynamics of fluids in porous media. Dover, New York, Elsevier, pp.19-20.

3. Berg, R.R., 1975. Capillary pressure in stratigraphic traps. AAPG Bull. 59(6), 939-959.

4. Bob, M. M., Brooks, M. C., Mravik, S.C., and Wood, A.L., 2008. A modified light transmission visualization method for DNAPL saturation measurements in 2-D models. Adv. Water Resour. 31(5), 727-742.

5. Bsavaraj, M.G., Gupta, G.S., Naveen, K., Rudolph, V. and Bali, R., 2005. Local liquid holdups and hysteresis in a 2-D packed bed using X-ray radiography, AIChE 51(8), 2178-2189.

6. Catalan, L., Xiao, W.F., Chatzis, I., and Dullien, F. A.L.,1992. An experimental study of secondary oil migration. AAPG Bull. 76(5), 638-650.

7. Chang, C.T., Mandava, S., Watson,. A.T., Sarker, S. and Edwards, C.M., 1993. The use of agarose gels for quantitative determination of fluid saturations in porous media. Magn. Reson. imaging 11(5), 717-725.

8. Chardaireriviere, C., and Roussel, J.C., 1991. Use of a high magnetic field to visualize fluids in porous media by MRI. paper SCA 9112.

9. Chen, S.H., Qin, F.F., Kim, K-H. Watson, A.T., 1992. NMR Imaging of multiphase flow in porous media. paper SPE 24670-MS presented at 1992 Annual Technical Conference and Exhibition, Washington, D.C., 4-7 October.

10. Conrad, S.H., Glass, R.J., and Peplinski, W.J., 2002. Bench-scale visualization of DNAPL remediation processes in analog heterogeneous aquifers: Surfactant floods and in situ oxidation using permanganate. J. Contam. Hydrol, 58, 13-49.

11. Darnault, C.J.G., Throop, J.A., DiCarlo, D.A., Rimmer, A., Steenhui, T.S., and Pralange, J.-Y., 1998. Visualization by light transmission of oil and water contents in transient two-phase flow fields. J. Contam. Hydrol. 31(3-4), 337-348.

12. Darnault, C.J.G., DiCarlo, D.A., Bauters, T.W.J., Jacobson, A.R., Throop, J.A., Montemagno, C.D., Parlange, J.-Y. and Steenhuis, T.S., 2001. Measurement of fluid contents by light transmission in transient three-phase oil-water-air systems in sand. Water Resour. Res. 37(7), 1859-1868.

13. Dembicki, H. J.R., and Anderson, M.J., 1989. Secondary migration of oil: Experiments supporting efficient movement of separate, buoyant oil phase along limited conduits. AAPG Bull. 73(8), 1018-1021.

14. Dicarlo, D.A., Bauters, T.W.J, Steenhuis, T.S., Parlange, J.-Y. and Bierck, B.R., 2000. High-speed 
measurements of three-phase flow using synchrotron X rays. Water Resour. Res. 33(4), 569-576.

15. Edelstein, W.A., Vinegar, H.J., Tutunjian, P.N., Roemer, P.B., and Mueller, O.M., 1988. NMR Imaging for Core Analysis, paper SPE 18272-MS presented at 1988 Annual Technical Conference and Exhibition, Houston, Texas, 2-5 October.

16. Enwere, M.P. and Archer, J.S., 1992. NMR Imaging for Water/Oil Displacement in Cores Under Viscous-Capillary Force Control. paper 24166-MS presented at 1992 SPE/DOE Enhanced Oil Recovery Symposium, Tulsa, Oklahoma, 22-24 April.

17. Fishman, M., Guarnaccia, J., Enfield, C., and Wood, L., 1998. DNAPL infiltration and distribution in subsurface: 2D experiment and modeling approach. First International Conference on Remediation of Chlorinated and Recalcitrant Compounds, VOL 2: Nonaqueous-Phase Liquids, pp. 37-42.

18. Frette, O.I., Måløy, K.J., Schmittbuhl, J. and Hansen, A., 1997. Immiscible displacement of viscosity matched fluids in two-dimensional porous media. Phys. Rev. E 55(3), 2969-2975.

19. Graue, A., Aspene,s E., Moe, R.W., Baldwin, B.A., Moradi, A., Stevens, J. and Tobola, D.P., 2001. MRI Tomography of saturation development in fractures during waterfloods at various wettability conditions, paper SPE 71506-MS presented at 2001 SPE Annual Technical Conference and Exhibition, New Orleans, Louisiana, 30 September-3 October.

20. Groesel, M., Gfoehler, M. and Peham, C., 2009. Alternative solution of virtual biomodeling based on CT-scans. J. Biomech. 42(12), 2006-2009.

21. Harms, J.C., 1966. Stratigraphic traps in a valley fill, western Nebraska. AAPG Bull. 50, 2119-2149.

22. Hill, E.H., III, Kupper, L.L., and Miller, C.T., 2002. Evaluation of path-length estimators for characterizing multiphase systems using polyenergetic X-ray absorptiometry. Soil Sci. 167(11), 703-719.

23. Hirsch, L.M. and Thompson, A.H., 1995. Minimum saturation and buoyancy in secondary migration. AAPG Bull. 79, 696-710.

24. Hoa, MT., 1981. A new method allowing the measurement of rapid variations of the water content in sandy porous media. Water Resour. Res. 17(1), 41-48.

25. Høst-Madsen, J. and Jensen, K.H., 1992. Laboratory and numerical investigations of immiscible flow in soil. J. Hydrol. 135(1-4),13-52.

26. Hou, P., Zhou, B., and Luo, X.R., 2004. Analysis on Patterns of Secondary Hydrocarbon Migration Pathways. Science in China (Volume D - Geoscience) 34 (Supplement 1), 162-168

27. Illangasekare, T.H., Ramsey, J.L., Jenson, K.H. and Butts, M., 1995. Experimental study of movement and 
distribution of dense organic contaminants in heterogeneous aquifers. J. Contam. Hydrol. 20(1-2), 1-25.

28. Imhoff, P.T., Thyrum, G.P., and Miller, C.T., 1996. Dissolution fingering during the solubilization of nonaqueous phase liquids in saturated porous media: 2. Experimental observations. Water Resour. Res. 32(7), 1929-1942.

29. Kenyon, W. E., 1992. Nuclear megnatic resonance as a petrophysical measurement. Nuclear Geophysics 6(2), 153-157.

30. Li, L.Q., Marica, F., Chen, Q., MacMillan, B. and Balcom, B.J., 2007. Quantitative discrimination of water and hydrocarbons in porous media by magnetization prepared centric-scan SPRITE. J. of Magn. Reson. $186(2), 282-292$.

31. Liu, Y.P., Steenhuis, T.S., Parlange, J.-Y., Bierck, B.R., and Selker, J.S., 1992. High intensity X-ray and tensiometer measurements in rapidly changing preferential flow fields. Soil Sci. Soc. Am. J. 57(5), 1188-1192.

32. Løvoll, G., Méheust, Y. , Toussaint, R., Schmittbuhl, J. and Måløy, K.J., 2004. Growth activity during fingering in a porous Hele Shaw cell. Phys. Rev. E. 70(2), 026301.

33. Luo, X., Zhang, F., Miao, S., Wang, W., Huang, Y., Zhou, B., Loggia, D. and Vasseur, G., 2004. Experimental verification of oil saturation and loss during secondary migration. J. Petrol. Geol. 27(3), 241-251.

34. Luo, X. R., Zhou, B., Zhao, S.X., Zhang, F.Q. and Vasseur, G., 2007. Quantitative estimates of oil losses during migration. Part I: the saturation of pathways in carrier beds. J. Petrol. Geol. 30(4), 375-387.

35. Luo, X. R., Yan, J. Z., Zhou, B., Wang, W., and Vasseur, G., 2008. Quantitative estimates of oil losses during migration, Part II: measurement of residual oil saturation in migration pathways. J. Petrol. Geol. 31(2), 179-190.

36. Mandava, S.S., Watson, T. A. and Edwards, C.M., 1990. NMR imaging of saturation during immiscible displacements. AIChE 36(11), 1680-1686.

37. McNeal, R.P., 1961. Hydrodynamic entrapment of oil and gas in Bisti field, San Juan County, New Mexico. AAPG Bull. 45, 315-329.

38. Méheust, Y., Løvoll, G., Måløy, K.J. and Schmittbuhl, J., 2002. Interface scaling in a two-dimensional porous medium under combined viscous, gravity and capillary effects. Phys. Rev. E 66(5), 051603.

39. Miao, S., Zhang, F.Q., Li, T.J., Luo, X.R. and Hou, P., 2004. Application of NMR Imaging Technique to Quantitative Observation and Analysis on Hydrocarbon Migration Pathway. Acta Petrolei Sinica 25(3), 44-47. 
40. Mogensen, K., Stendy, E. And Zhou, D., 2005. Studies of waterflooding in low-permeable chalk by use of X-ray CT Scanning. J. Petrol. Sci. Eng. 32(1), 1-10.

41. Nakashima, Y. and Kamiya, S., 2007. Programs for the analysis of three-dimensional pore connectivity and anisotropic tortuosity of porous rocks using X-ray computed tomography image data. J. Nucl. Sci. Technol. 44(7), 1233-1247.

42. Nicula, S., Brancolini, A., Cominelli, A., and Mantica, S., 1998. Blending magnetic resonance imaging and numerical simulation. paper SCA 9819.

43. O'Carroll, D.M., Bradford, S.A., and Abrioba, L.M., 2004. Infiltration of PCE in a system containing spatial wettability variations. J. Contam. Hydrol. 73(1-4), 39-63.

44. Oostrom, M., Dane, J.H., and Wietsma, T.W., 2005. Removal of carbon tetrachloride from a layered porous medium by means of soil vapor extraction enhanced by desiccation and water table reduction. Vadose Zone J. 4, 1170-1182.

45. Oostrom, M., Hofstee, C., Lenhard, R.J. and Wietsma, T.W., 2003. Flow behavior and residual saturation formation of injected carbon tetrachloride in unsaturated heterogeneous porous media. J. Contam. Hydrol. 64(1-2), 93-112.

46. Pape H., Arnold J. and Pechnig R. Clauser, C., Talnishnikh, E., Anferova, S. and Blümich E., 2009. Permeability prediction for low porosity rocks by mobile NMR. Pure appl. Geophys 166, 1125-1163.

47. Rangel-German, E., Akin, S. And Castanier, L., 2006. Multiphase-flow properties of fractured porous media. J. Petrol. Sci. Eng. 51(3-4), 192-213.

48. Schowalter, T.T., 1979. Mechanics of secondary hydrocarbon migration and entrapment. AAPG Bull. 3(5), 723-760.

49. Sharma, B.C., Brigham, W.E. and Castainier, L.M., 1997. CT imaging techniques for two-phase and three-phase in-situ saturation measurements. report SUPRI TR 107, Stanford University.

50. Smith, D.A., 1966. Theoretical considerations of sealing and non-sealing faults. AAPG Bull. 50, 363-374.

51. Tang, H.J., and Cui, K.H., 2007. Reservoir Physics. Petroleum industry, Beijing, pp. 89-94.

52. Toussaint, R., Løvoll, G., Méheust, Y., Måløy, K.J. and Schmittbuhl, J., 2005. Influence of pore-scale disorder on viscous fingering during drainage, Europhys. Lett., 71(4), 583.

53. Tukunaga, T., Mogi, K., Matsubara, O., Tosaka, H. and Kojima, K., 2000. Buoyancy and interfacial force effects on two phase displacement patterns: an experimental study. AAPG Bull. 84(1), 65-74.

54. Ursin, J.R., Detection of fluid saturation levels in porous media using gamma-ray tomography. J. Petrol. Sci. 
Eng. 7(3-4), 197-308.

55. Van Geel, P.J., and Sykes, J.F., 1994. Laboratory and model simulations of a LNAPL spill in a variably-saturated sand: 1.Laboratory experiment and image analysis techniques. Contam. Hydrol. 17, 1-25.

56. Wang, H.Z, Zhang, X.1., and Wu, J., 2008. Magnetic resonance imaging test tutorial. China science, Beijing, pp. $90-95$

57. Washburn, K. E. and Madelin, G., 2010. Imaging of multiphase fluid saturation within a porous material via sodium NMR. J. of Magn. Reson. 202(1), 122-126

58. Zeng, J.H., and Wang, J., 2003. Mechanisms and Physical Simulation of Hydrocarbon Migration. Petroleum industry, Beijing, pp. 1-187.

59. Zhang, F.Q., Luo, X.R., Miao, S., Wang, W.M., Zhou, B. and Huang, Y.Z., 2003. Patterns and Influencing Factors of Secondary Hydrocarbon Migration. Petroleum Experiment and Geology 25(1), 69-75.

60. Zhang, F.Q., Luo, X.R., Miao, S., Wang, W.M., and Zhou, B., 2004. Experiments on Forming Process of Dominant Path for Secondary Hydrocarbon Migration \& Mechanisms Analysis. Chinese Journal of Geology 39(2), 159-167.

61. Zhang, Y.S., Zhao, Z.F. and Chen, F.M., 2003. DIB Display Technique for DICOM-based Medical Images. Application and Research of Computers 2, 75-76

62. Zhou, B., Hou, P., Wang, W.M. and Luo, X.R., 2005. Oil Saturation Analysis in Migration Passways with NMR Imaging Technique. Petroleum Exploration and Development 32(6), 78-81. 
1 Figure 1 Water transverse relaxation time as a function of $\mathrm{Mn}^{2+}$ concentration in tested porous medium

2 Figure 2 MRI slice (cross section at the middle of the tube) and gray level distribution (In Figure 2A, the 3 pixel size is $\left.1 \mathrm{~mm}^{*} 1 \mathrm{~mm}\right)$

4 Figure 3 Effect of window widths and levels on the image format conversion from DICOM to BMP. A:

5 window and level were adjusted to make the migration path clearest; B: the window width covering the 6 whole signal ranges. Figure 4 Effect of window widths and levels on oil distribution. Oil saturation in Figure 4A and 4B were calculated according to figure $3 \mathrm{~A}$ and $3 \mathrm{~B}$ respectively.

Figure 5 Oil Saturation distribution calculated in DICOM image format directly.

Figure 6 Comparison of the actually injected oil volume and the calculated total oil volume. The whole migration path was scanned again once the path front migrated upwards $5 \mathrm{~cm}$.

Figure 7 Oil saturation distribution in finger migration path at the middle of the tube.

Figure 8 Oil saturation distribution in piston migration path at the middle of the tube.

Figure 9 Effect of MRI resolutions on the distribution curves of MRI signal intensity as a function of position

Table 1 Comparison between saturation measurement methods

Table 2 Relaxation time of oil in glass bead pores of different grain sizes $\mathbf{r}$ 
Table 1

\begin{tabular}{c|c|c|c|c|c|c|c|c|c}
\hline $\mathrm{r}(\mathrm{mm})$ & 0.08 & $0.2-0.4$ & $0.4-0.6$ & $0.6-0.8$ & $0.8-1.0$ & $1.0-1.2$ & $1.5-2.0$ & $2.0-2.5$ & 0 \\
\hline$T_{2}$ (ms) & 294 & 668 & 810 & 868 & 920 & 956 & 987 & 1028 & 1151 \\
\hline
\end{tabular}

Grain size of zero represents a pure oil sample

Table 2

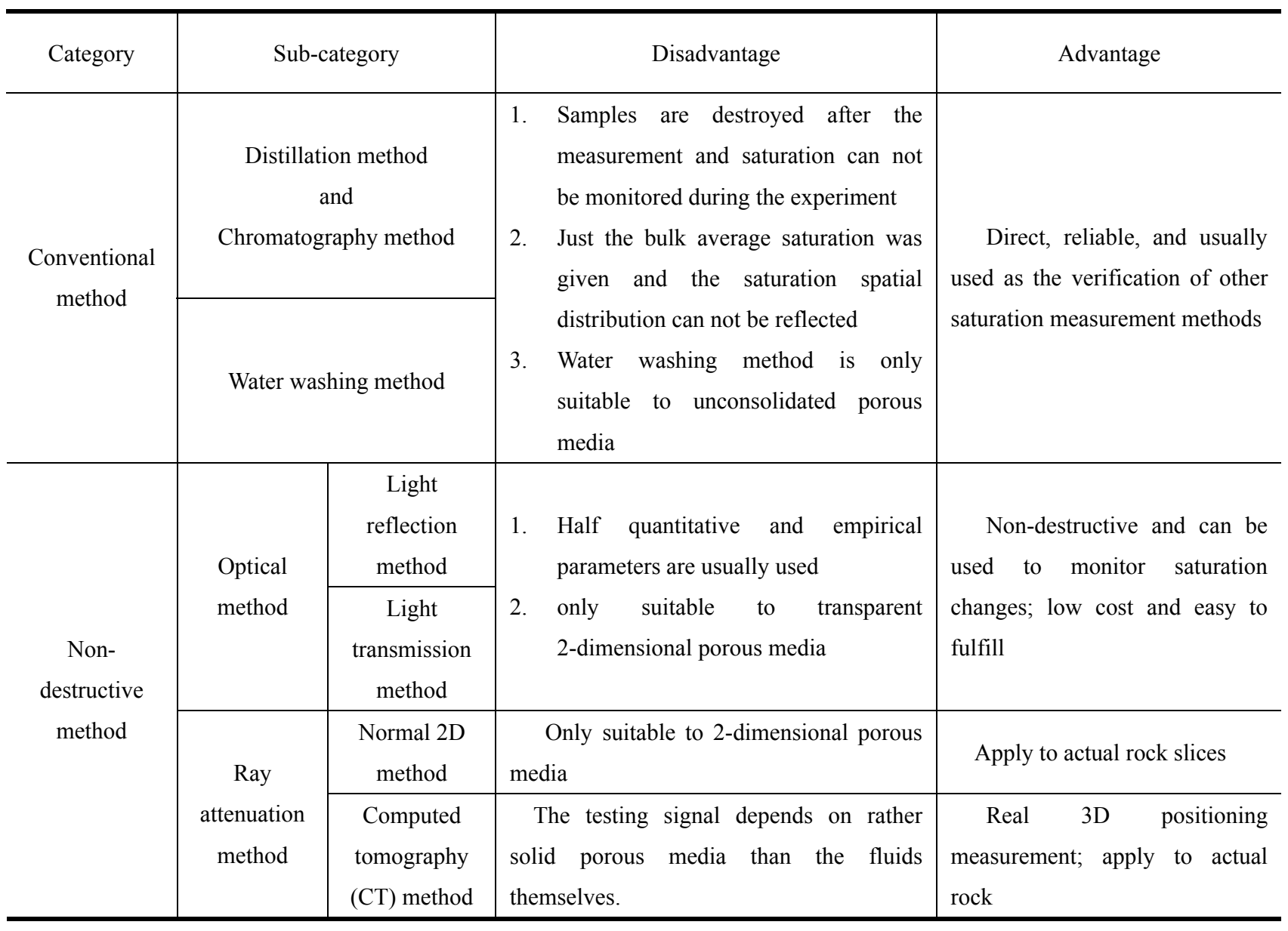




\section{Figure 1}

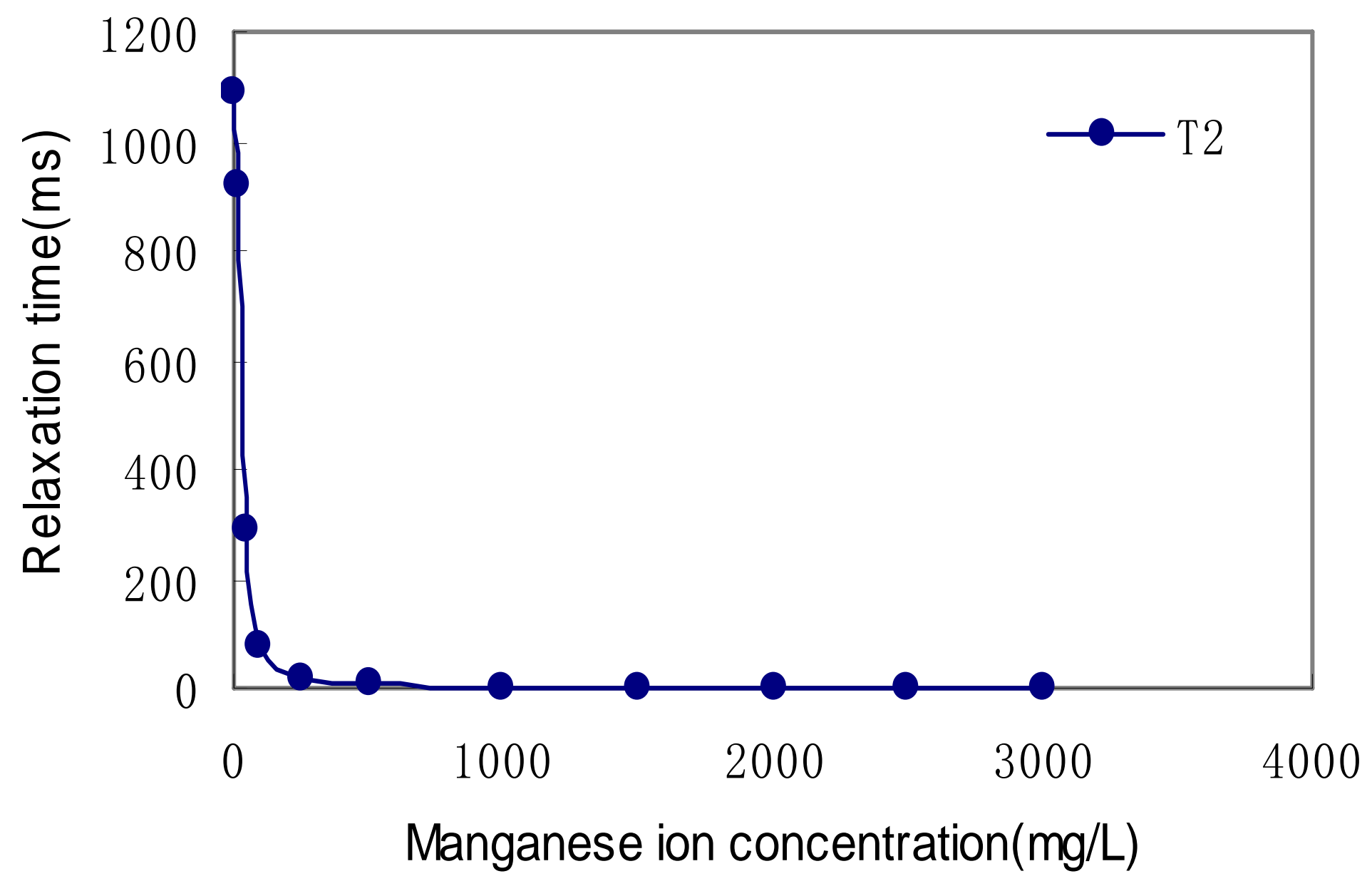




\section{Figure 2}

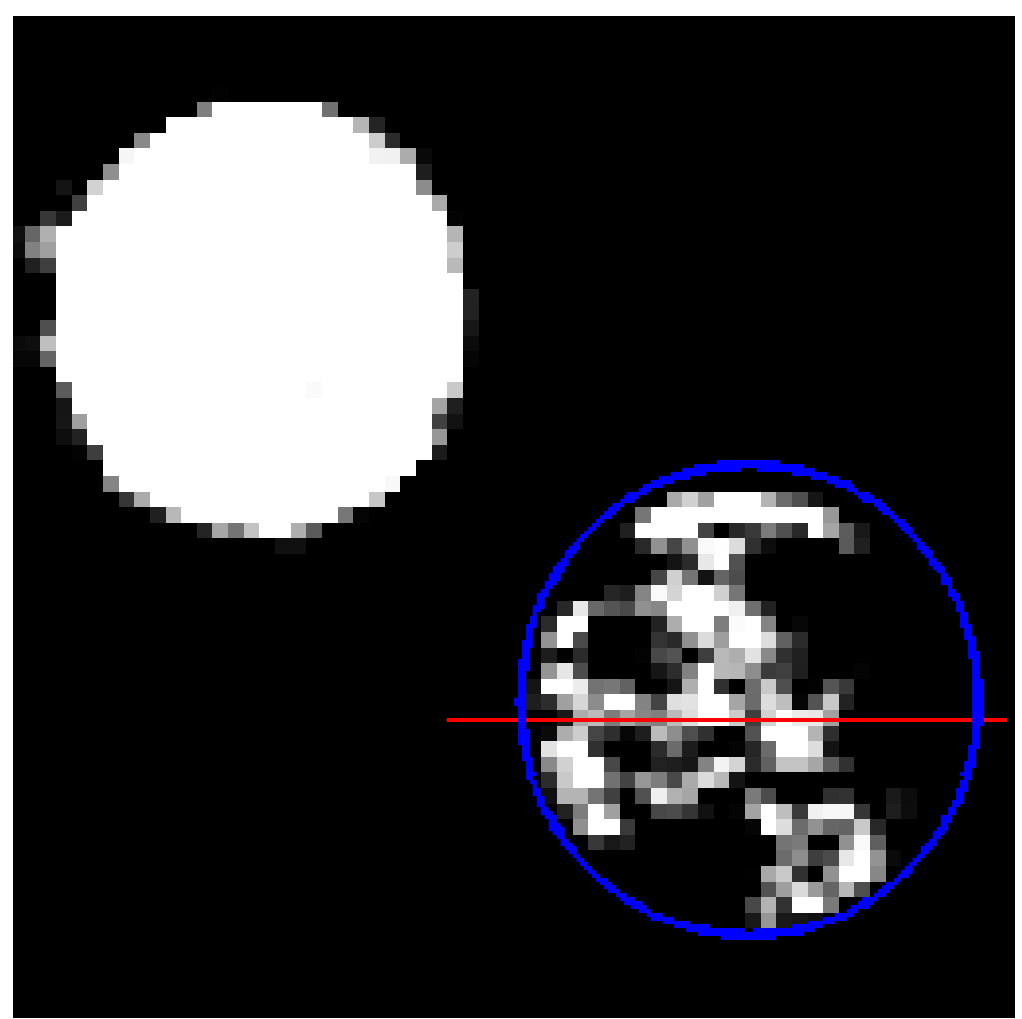

A. MRI Slice

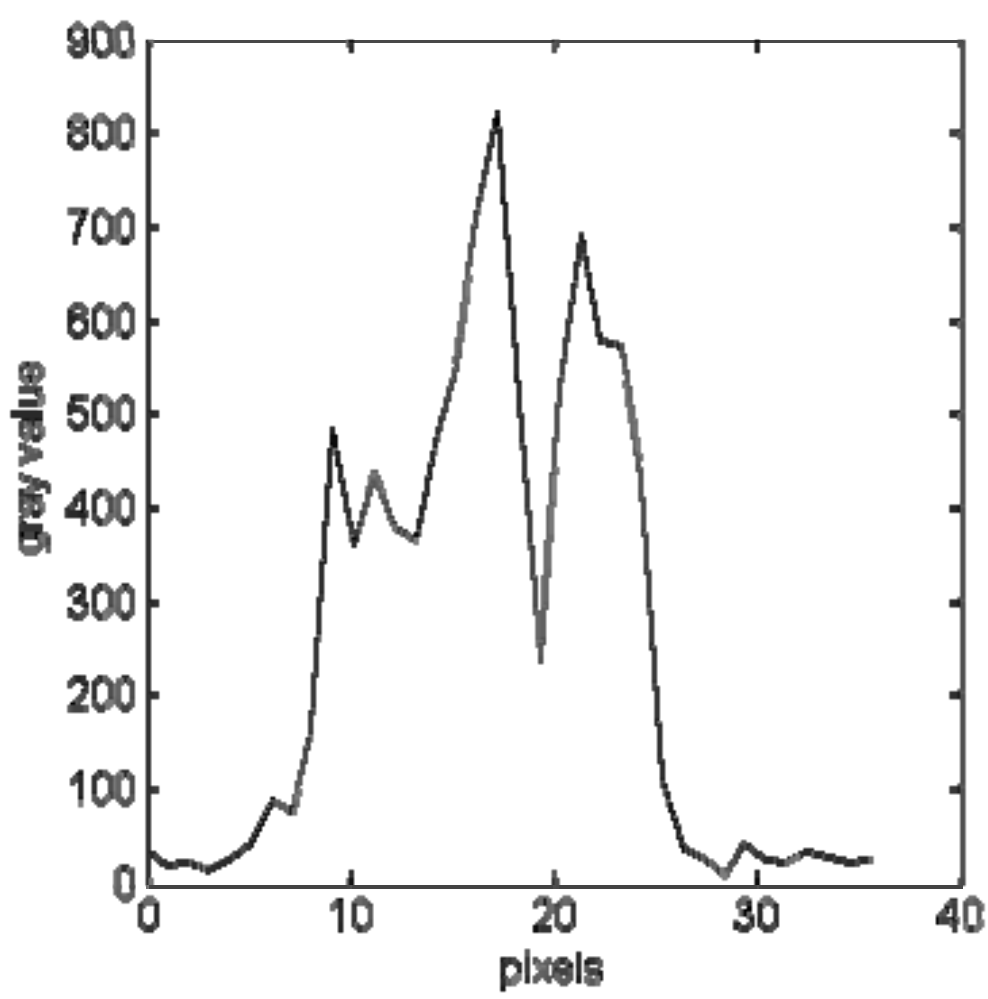

B. Gray Level Distribution Curve 


\section{Figure 3}

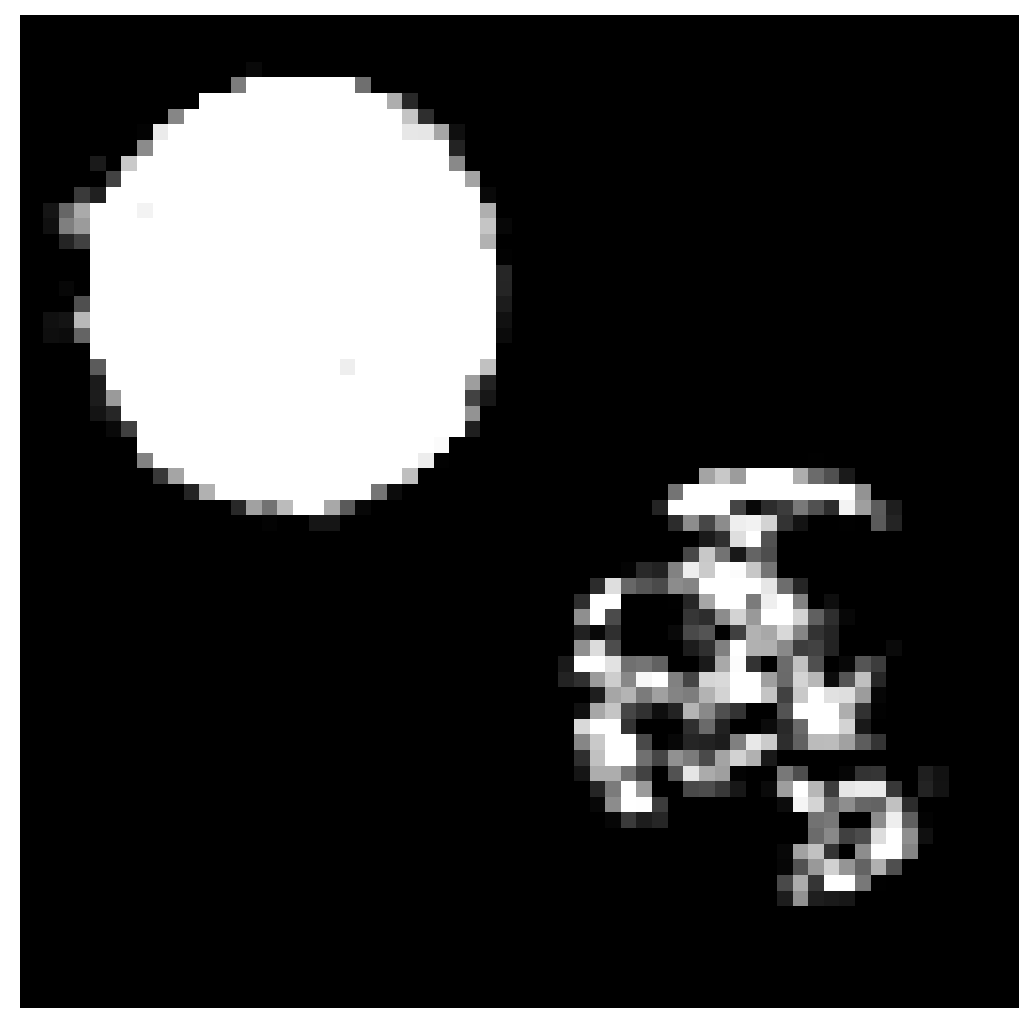

A

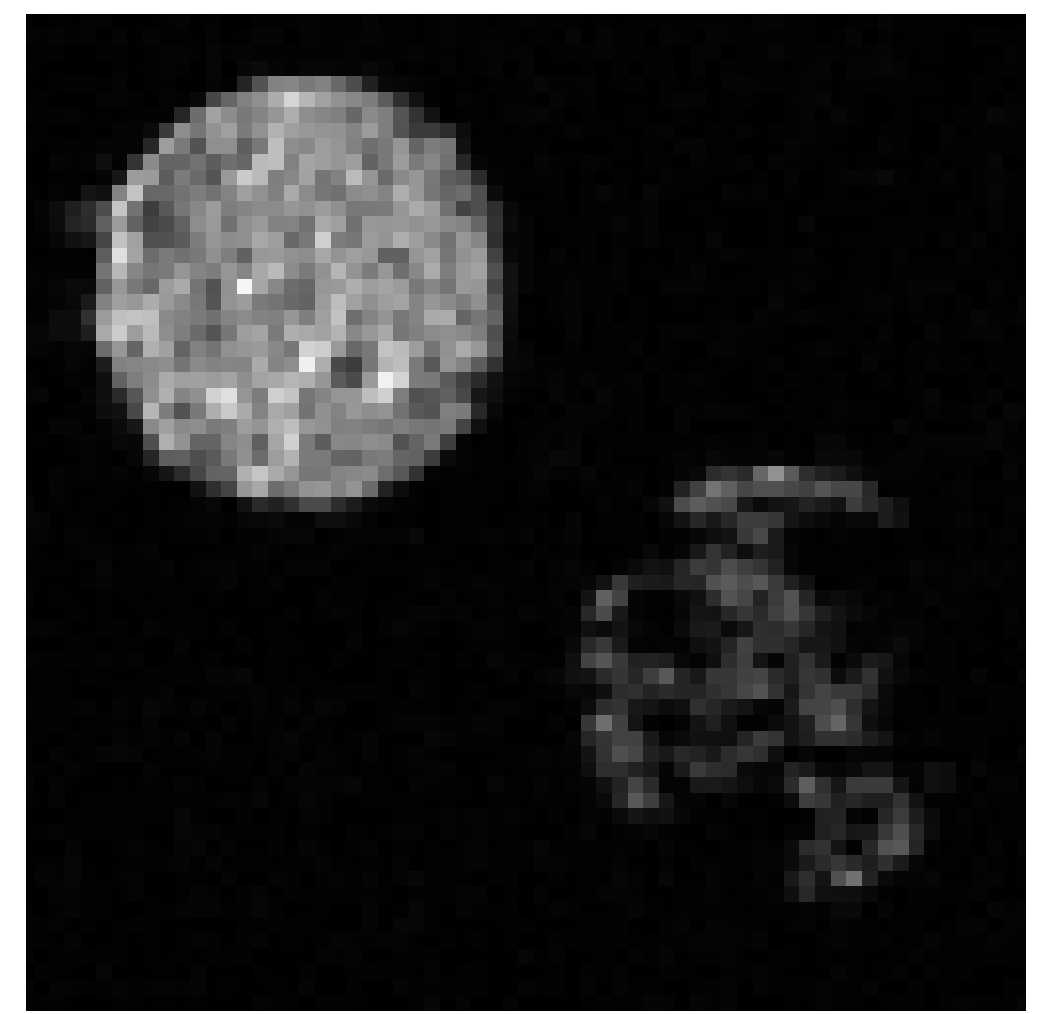

B 


\section{Figure 4}

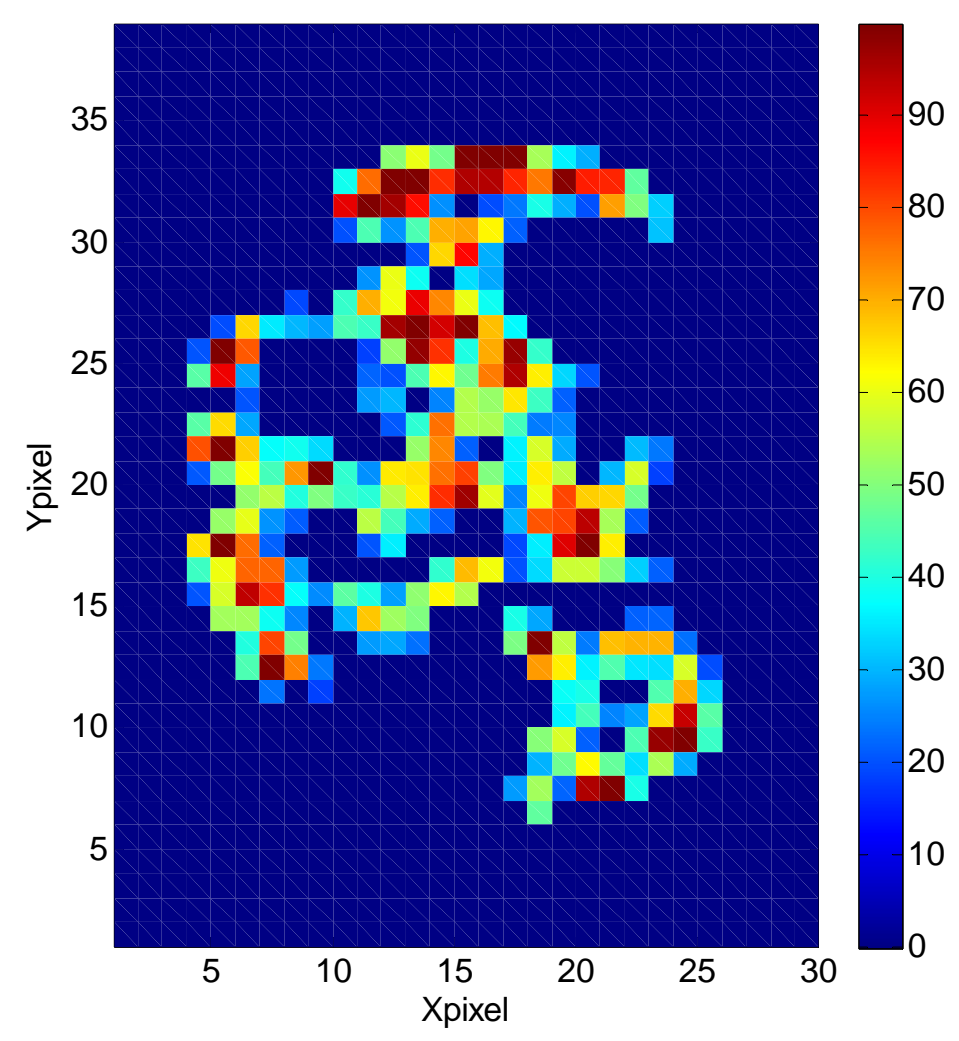

A

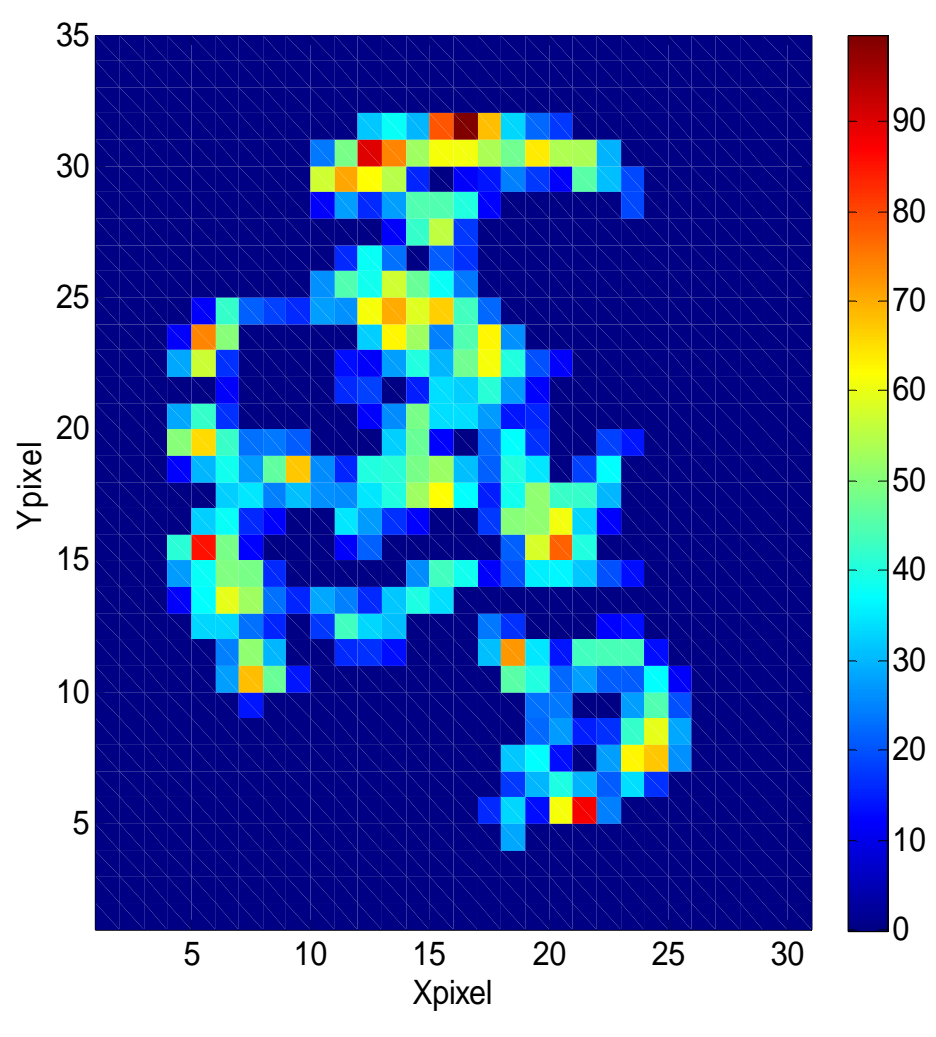

B 


\section{Figure 5}

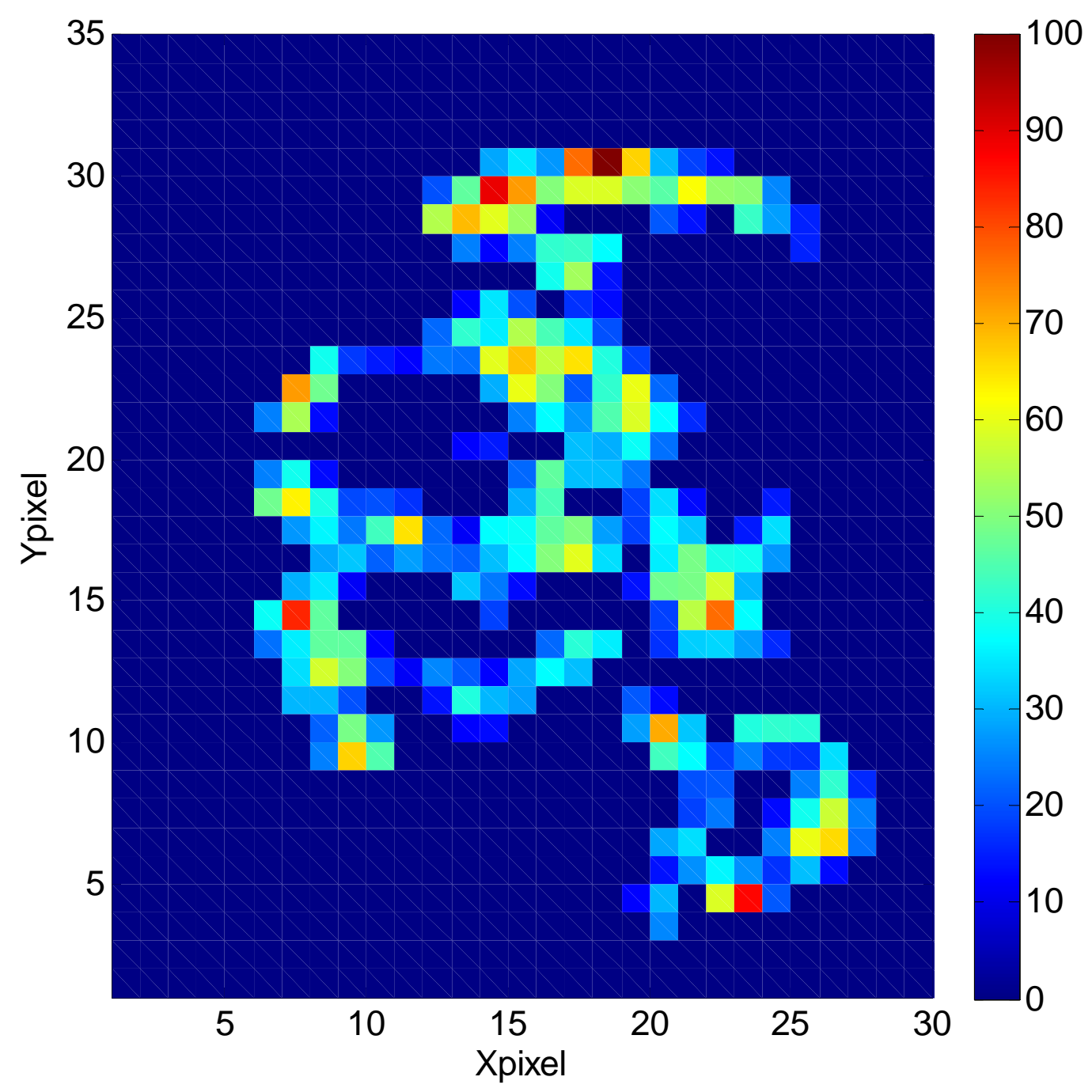




\section{Figure 6}

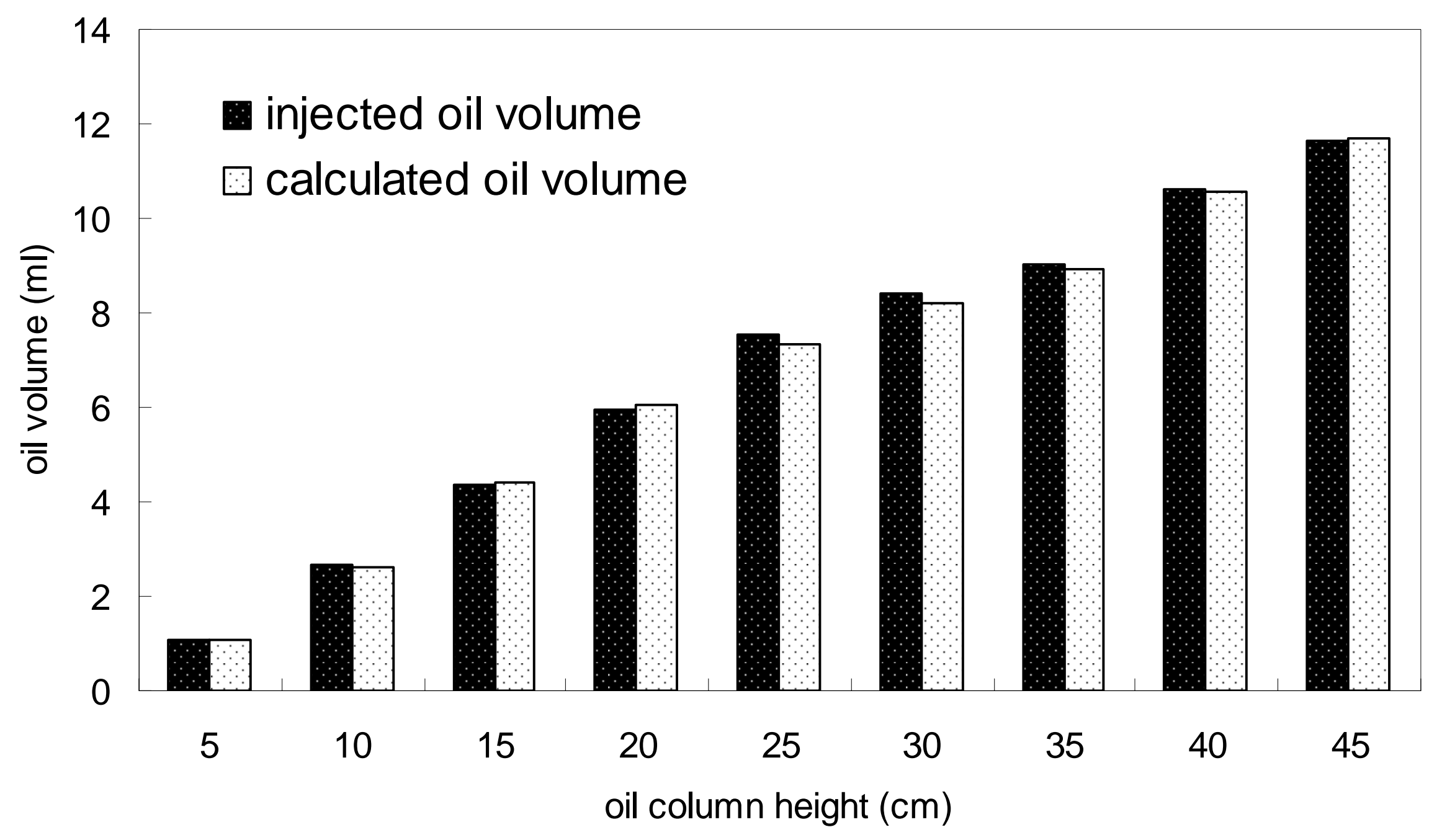




\section{Figure 7}

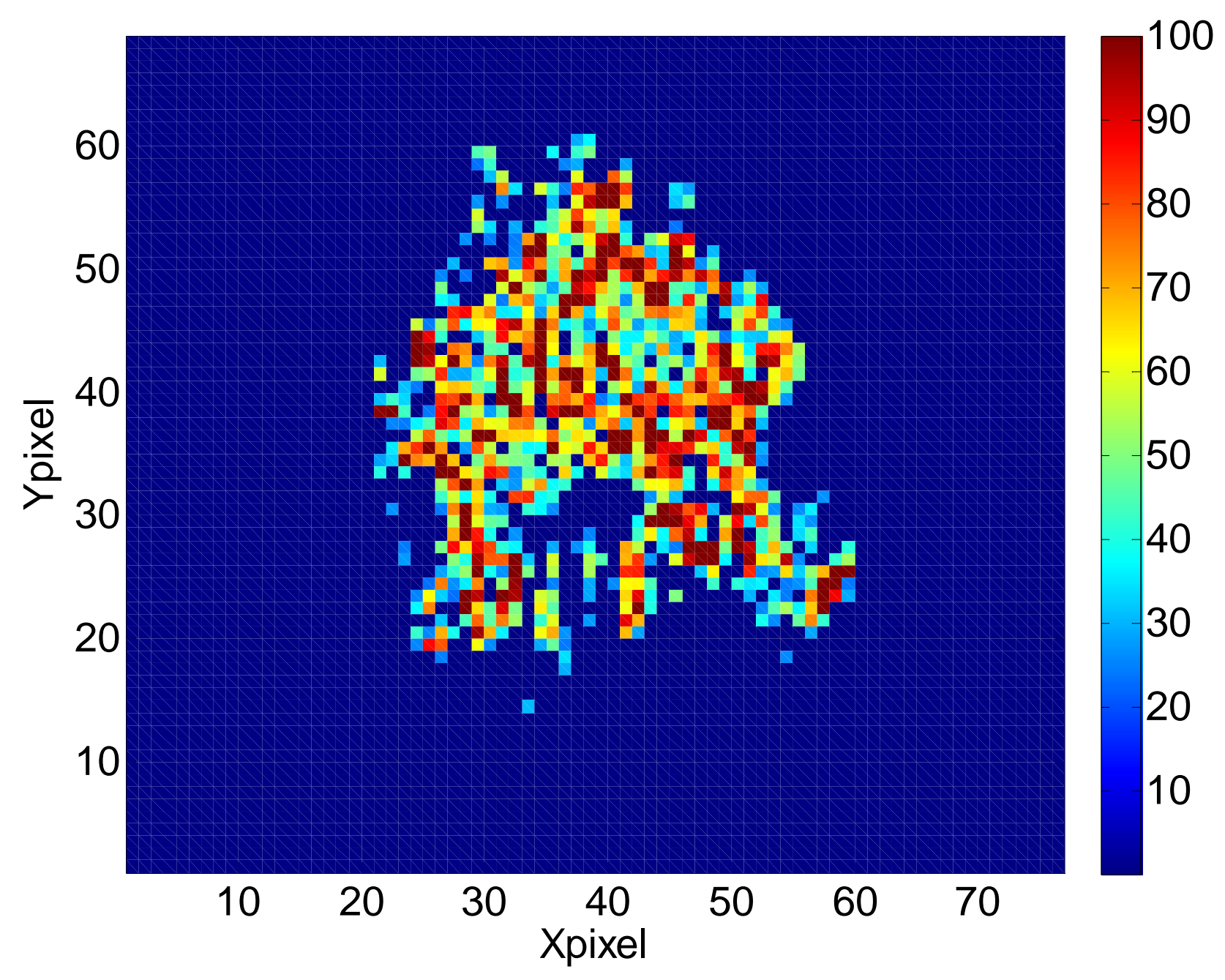




\section{Figure 8}

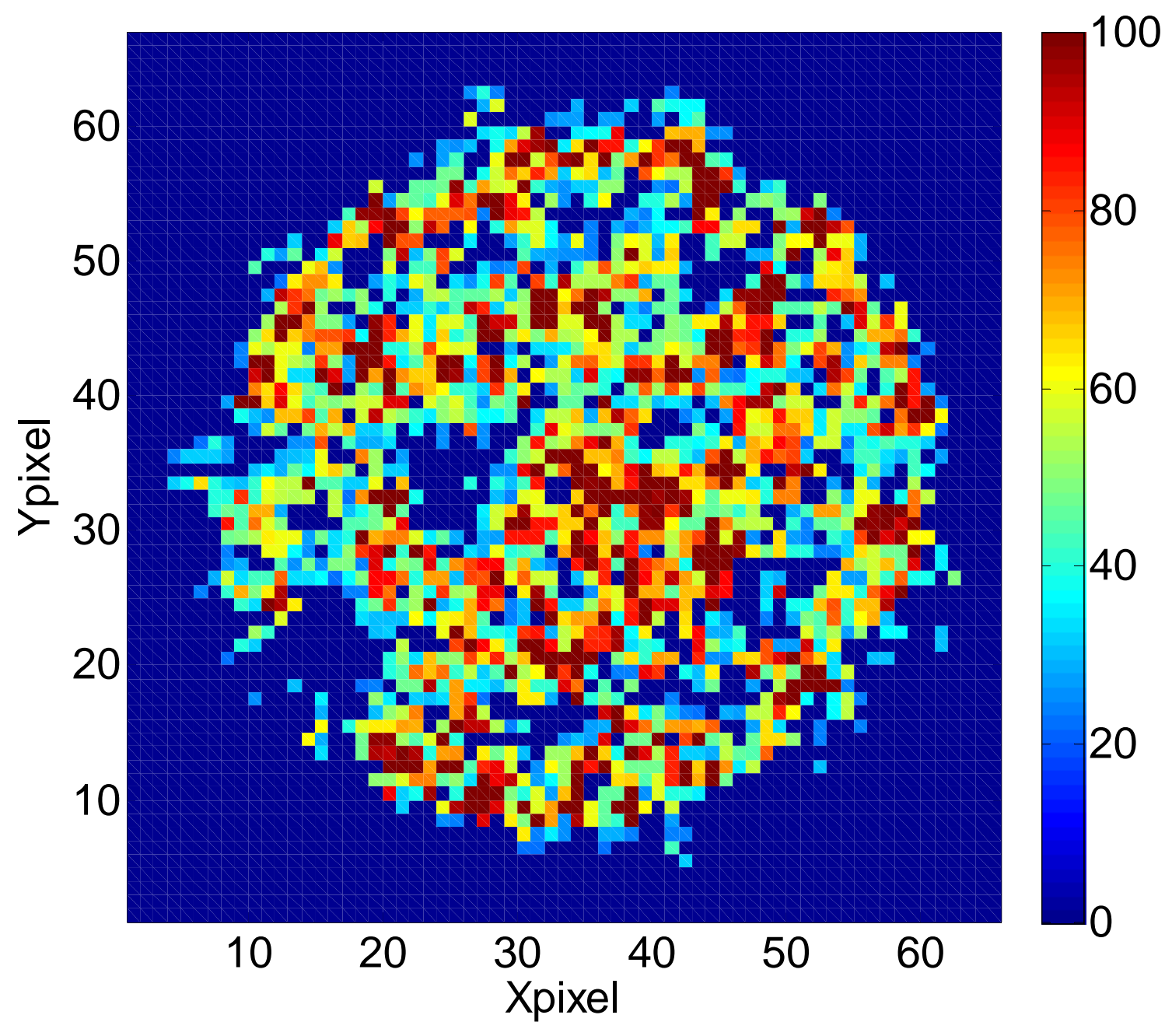




\section{Figure 9}

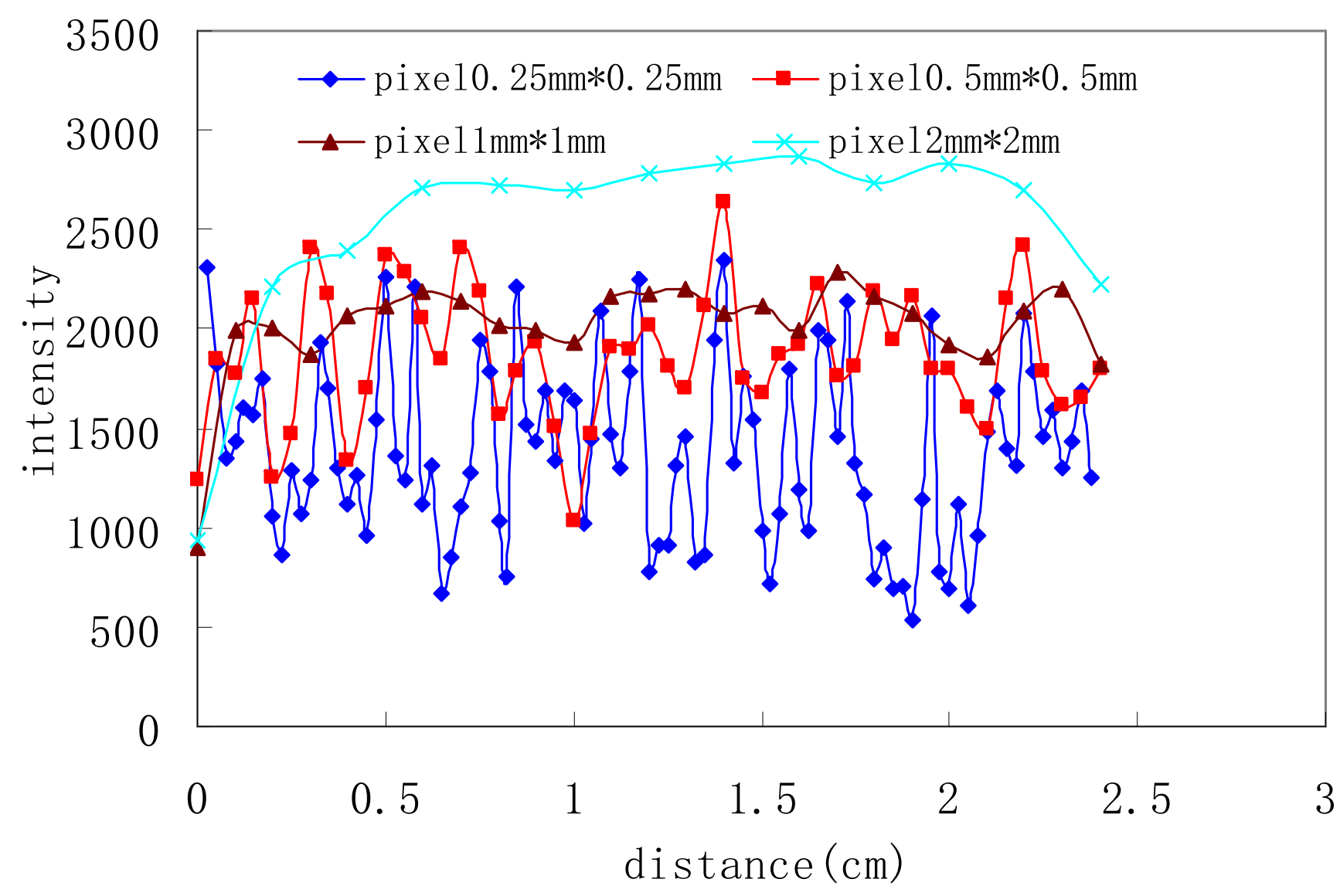

